\title{
Preclinical Pharmacokinetic/Pharmacodynamic Modeling and Simulation in the Pharmaceutical Industry: An IQ Consortium Survey Examining the Current Landscape
}

\author{
Edgar Schuck, ${ }^{1,19}$ Tonika Bohnert, ${ }^{2}$ Arijit Chakravarty, ${ }^{3}$ Valeriu Damian-Iordache, ${ }^{4}$ Christopher Gibson, ${ }^{5}$ \\ Cheng-Pang Hsu, ${ }^{6}$ Tycho Heimbach, ${ }^{7}$ Anu Shilpa Krishnatry, ${ }^{4}$ Bianca M Liederer, ${ }^{8}$ Jing Lin, ${ }^{9}$ Tristan Maurer, ${ }^{10}$ \\ Jerome T Mettetal, ${ }^{11}$ Daniel R Mudra, ${ }^{12}$ Marjoleen JMA Nijsen, ${ }^{13}$ Joseph Raybon, ${ }^{14}$ Patricia Schroeder, ${ }^{15}$ \\ Virna Schuck, ${ }^{11,18}$ Satyendra Suryawanshi, ${ }^{14}$ Yaming Su, ${ }^{16}$ Patrick Trapa, ${ }^{10}$ Alice Tsai, ${ }^{17}$ Majid Vakilynejad, ${ }^{3}$ \\ Shining Wang, ${ }^{16}$ and Harvey Wong ${ }^{8,19}$
}

Received 13 November 2014; accepted 26 December 2014; published online 29 January 2015

\begin{abstract}
The application of modeling and simulation techniques is increasingly common in preclinical stages of the drug discovery and development process. A survey focusing on preclinical pharmacokinetic/ pharmacodynamics (PK/PD) analysis was conducted across pharmaceutical companies that are members of the International Consortium for Quality and Innovation in Pharmaceutical Development. Based on survey responses, $\sim 68 \%$ of companies use preclinical PK/PD analysis in all therapeutic areas indicating its broad application. An important goal of preclinical PK/PD analysis in all pharmaceutical companies is for the selection/optimization of doses and/or dose regimens, including prediction of human efficacious doses. Oncology was the therapeutic area with the most PK/PD analysis support and where it showed the most impact. Consistent use of more complex systems pharmacology models and hybrid physiologically based pharmacokinetic models with PK/PD components was less common compared to traditional PK/PD models. Preclinical PK/PD analysis is increasingly being included in regulatory submissions with $\sim 73 \%$ of companies including these data to some degree. Most companies ( $86 \%)$ have seen impact of preclinical PK/PD analyses in drug development. Finally, $\sim 59 \%$ of pharmaceutical companies have plans to expand their PK/PD modeling groups over the next 2 years indicating continued growth. The growth of preclinical PK/PD modeling groups in pharmaceutical industry is necessary to establish required resources and skills to further expand use of preclinical PK/PD modeling in a meaningful and impactful manner.
\end{abstract}

KEY WORDS: pharmaceutical industry; preclinical pharmacokinetic/pharmacodynamic modeling; simulation.

\footnotetext{
${ }^{1}$ Modeling and Simulation, Eisai Inc., 155 Tice Blvd, Woodcliff Lake, NJ 07677, USA.

${ }^{2}$ Biogen-Idec, Cambridge, MA, USA.

${ }^{3}$ Takeda, Deerfield, iL, USA.

${ }^{4}$ GlaxoSmithKline, Upper Marion, PA, USA.

${ }^{5}$ PPDM, Merck Research Laboratories, West Point, PA, USA.

${ }^{6}$ PKDM, Amgen Inc., Thousand Oaks, CA, USA.

${ }^{7}$ Novartis Institutes for Biomedical Research, East Hanover, NJ, USA.

${ }^{8}$ Drug Metabolism and Pharmacokinetics, Genentech Inc., 1 DNA Way, MS 412a, South San Francisco, CA 94080, USA.

${ }^{9}$ Sunovion, Marlborough, MA, USA.

${ }^{10}$ Pfizer Inc., Cambridge, MA, USA.

${ }^{11}$ Drug Safety and Metabolism, Astra-Zeneca, Waltham, MA, USA.

${ }^{12}$ Eli Lilly and Company, Indianapolis, IN, USA.

${ }^{13}$ Abbvie, North Chicago, IL, USA.

${ }^{14}$ Bristol-Myers Squibb, Princeton, NJ, USA.

${ }^{15}$ EMD Serono, Billerica, MA, USA.

${ }^{16}$ Sanofi, Bridgewater, NJ, USA.

${ }^{17}$ Vertex Pharmaceuticals Incorporated, Boston, MA, USA.

${ }^{18}$ Present Address: Novartis, East hanover, NJ, USA.

${ }^{19}$ To whom correspondence should be addressed. (e-mail: Edgar_Schuck@eisai.com; wong.harvey@gene.com)
}

\section{INTRODUCTION}

Drug discovery and development has been plagued by clinical attrition rates exceeding $90 \%$, where the primary cause was identified by Kola and Landis (6) as lack of efficacy and unexpected safety findings. The authors highlighted that, while poor pharmacokinetics was identified as a main cause for drug attrition in the early 1990s, this was no longer a leading cause for attrition 10 years later. Advances in preclinical in vitro $(9,5,10)$ and in vivo methodologies $(13$, $14,5,10)$ for predicting human pharmacokinetics served to reduce the drug failure rate due to poor pharmacokinetics such that, by 2000 , lack of efficacy became the main cause of drug attrition. In an updated look at causes for drug failures in phase II (between 2008 and 2010; (1)) and phase III and drug submission (between 2007 and 2010; (2)), lack of efficacy remained the primary cause of attrition contributing to $51 \%$ and $66 \%$ of drug failures, respectively.

The International Consortium for Innovation and Quality in Pharmaceutical Development (IQ Consortium) is an organization of pharmaceutical and biotechnology companies with the mission of advancing science-based and 
scientifically driven standards and regulations for pharmaceutical and biotechnology products worldwide. The need to improve an understanding of the drivers of efficacy was recently highlighted at the 2013 IQ Consortium Drug Metabolism Leadership Group managers meeting. In a survey conducted at the meeting, $>90 \%$ of the attendees agreed that an "in-depth understanding of drivers of efficacy, including biomarkers and translational sciences" would lead to the greatest advances in drug discovery and development over the next 15 years. Pharmacokinetic/ pharmacodynamic (PK/PD) modeling and simulation is an important tool that can provide a better understanding of drug efficacy at an earlier stage in the drug discovery and development process.

$\mathrm{PK} / \mathrm{PD}$ analysis involves the use of mathematical functions to quantitatively interrelate drug behavior and its' effects on the biological system of interest. This type of analysis is particularly helpful in understanding exposureresponse relationships where there are apparent disconnects in drug concentration and effect related to temporal delays in drug action. In addition, mechanism-based PK-PD models allow the quantitative assessment of known species differences in factors such as pharmacokinetics or in vivo drug potency, and enable a more robust characterization of concentration-pharmacodynamic/effect relationships from frequently variable in vivo preclinical efficacy data. More recently, there has been an increase in reports describing the utility of PK/PD analysis to help interpret and translate the results of preclinical studies to human $(11,3,7,8,15-17,4$, 18). Finally, the establishment of PK/PD models enables integration of all available data and allows for leveraging of these data for prospective simulations. Just as an early assessment of preclinical pharmacokinetics helped to reduce drug failures due to poor human pharmacokinetics in the 1990s, a better understanding of drug effect using preclinical pharmacokinetic/pharmacodynamic analysis offers an important tool to potentially reduce drug attrition due to a lack of efficacy.

The IQ PK/PD Discussion Group (PK/PDDG), consisting of representatives from 17 pharmaceutical companies, was formed by the IQ Consortium Drug Metabolism Leadership Group as a means to promote innovation through sharing of best practices involved with preclinical PK/PD analysis. The PK/ PDDG identified a clear need to better understand the landscape of preclinical PK/PD analysis in pharmaceutical industry. Therefore, a survey focusing on preclinical PK/PD analysis was conducted across IQ member companies. This communication presents the survey results and attempts to provide insights on the current state of preclinical PK/PD analysis in pharmaceutical industry including barriers that may impede its broad use in an impactful manner.

\section{METHODS}

The results presented in this communication were obtained by conducting an industry wide survey of pharmaceutical companies that are members of the IQ Consortium. Survey questions were provided and edited to their final form by members of the IQ Consortium PK/PDDG. The types of questions included single/multiple choice, dichotomous (e.g., yes/no), rank questions, and open-ended or free text. The final survey consisted of 49 questions aimed to gather information in five different areas: demographics, organizational structure, operating logistics, applications, and impact/ perspectives. In some instances, certain questions had a small degree of overlapping information but were collated in the area that provided the best context to the question. "Demographics" questions provided information about the general characteristics of the pharmaceutical companies and the composition of the groups responsible for preclinical PK/PD modeling. "Organizational structure" questions focused on gathering information regarding how preclinical PK/PD groups fit within the organizational structure of various pharmaceutical companies. "Operating logistics" questions provided information on practical operational aspects of preclinical PK/PD modeling groups. "Application" questions gathered information on how preclinical PK/PD modeling is being utilized in each company and in which therapeutic areas. Finally, "impact/perspectives" questions assessed how preclinical PK/PD modeling is perceived within each company, its place in the company's decision-making processes, its impact on project progression, and the challenges for implementation of preclinical PK/PD modeling throughout the drug discovery and development process.

The survey was conducted using an online questionnaire and survey software (SurveyMonkey; www.surveymonkey.com) and sent to representatives of IQ Consortium member companies that agreed to participate in the survey. Each representative was responsible for providing responses that were reflective of the company as a whole since only one response was collected from each company. The collection of responses occurred over an approximately 1-month period (October 17, 2013 to November 15, 2013). All responses to the questionnaire were kept anonymous. Data analysis and graphs were generated using Microsoft Excel v10 (Microsoft Corporation, Redmond, WA, USA). In some cases, data analysis was performed to see if company size impacted survey responses. Data analyses that considered company size compared pharmaceutical companies with $\leq 10,000$ employees (designated as small to mid-sized companies) to pharmaceutical companies with $>10,000$ employees (designated as large companies). This division of companies into two groups was due to the lower number of companies with $<10,000$ employees participating in the survey ( 7 of 22). Finally, the authors reviewed survey responses as a group and provided potential reasons as to why certain trends were observed. These thoughts have been included in the manuscript and may result in a certain level of bias in the discussion of survey results. However, the authoring group is a reasonable representation of individuals involved in preclinical PK/PD analysis in pharmaceutical industry so these opinions serve to provide context to survey results.

\section{RESULTS AND DISCUSSION}

Despite the perceived importance of $\mathrm{PK} / \mathrm{PD}$ in the discovery and development of new medicines, there have been a limited number of surveys assessing the current state of PK/PD modeling in pharmaceutical industry. A recent survey by Vlasakakis et al. (12) surveyed the landscape of drug and disease modeling and simulation in both academia and industry. However, the focus of that survey was to gather 
information to develop a framework of competencies to guide future training of $\mathrm{PK} / \mathrm{PD}$ scientists in both academia and industry. To our knowledge, the survey described in this manuscript serves as the first to specifically examine the state of preclinical PK/PD analysis in pharmaceutical industry. The survey results have been grouped into the following sections: demographics, organizational structure, operating logistics, applications, and impact/perspectives.

\section{Demographics}

Survey results were gathered over an approximate 1 month period during which responses were received from 22 pharmaceutical companies. The breakdown of the size of the pharmaceutical companies that participated in the survey is shown in Fig. 1.

The majority of the survey respondents were from companies with $>10,000$ employees $(\sim 68 \%)$. This is reflective of the composition of IQ Consortium membership, which consists largely of major pharmaceutical companies with research units based in the USA.

With the rise in the development of biologics by pharmaceutical companies, we wanted to gather information on whether or not a research focus on traditional small molecules vs biologics influenced the use of preclinical PK/PD modeling. Figure $2 \mathrm{a}$ shows that currently the majority of the US-based pharmaceutical companies (68\%) focus their efforts on both small molecules and biologics, which is reflective of the trend to diversify portfolios especially in large pharmaceutical companies. Based on the large proportion of companies with mixed small molecule and biologics focus, we were unable to examine the effect of research focus on the use of preclinical PK/PD modeling. Of interest, small to midsized pharmaceutical companies ( $\leq 10,000$ employees) appeared to work primarily on small molecules (71\%; Fig. 2b) compared to large pharmaceutical companies $(>10,000 \mathrm{em}$ ployees), which focused on both classes of therapies $(86 \%$; Fig. 2c). Such a trend may be related to a smaller amount of resources and expertise in small to mid-sized companies limiting diversification.

The breakdown of clinical and preclinical PK/PD modelers is shown in Fig. 3. Companies appeared to have more clinical modelers compared to preclinical modelers with $\sim 50 \%$ of the companies involved in the survey having $>10$ clinical modelers compared to $\sim 33 \%$ of companies having $>10$ preclinical modelers. No difference in this trend was observed when examining the small to mid-sized vs large companies. It is not possible to explain the reason for the difference in the number of preclinical vs. clinical modelers based on the responses of the survey. However, the greater

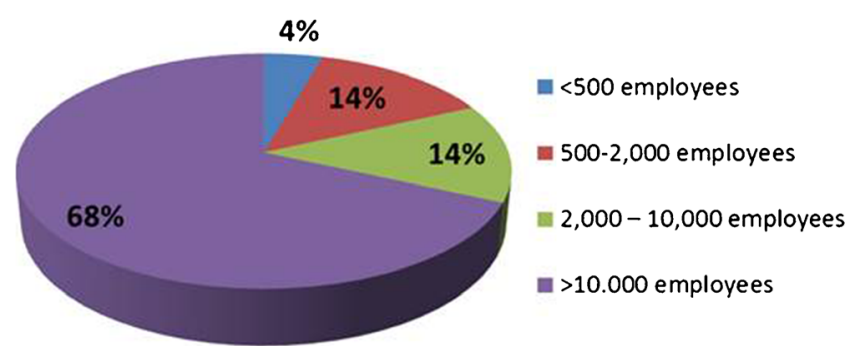

Fig. 1. Pie chart showing size of the pharmaceutical companies providing responses to survey number of clinical modelers may be reflective of an evolution of $\mathrm{PK} / \mathrm{PD}$ modeling from the clinical to preclinical area similar to what occurred during the implementation of pharmacokinetics in pharmaceutical industry. In addition, the nature of the work in the clinical $v s$ the preclinical space is different with analyses of clinical trial data often being more labor intensive and thus requiring more modelers. However, it appears that the majority of companies ( $59 \%)$ intend to increase the number of preclinical modelers in the next 2 years, highlighting the investment in preclinical modeling by IQ member companies (Fig. 3). The growth appears to be largely in large companies ( $>10,000$ people) with $73 \%$ of large companies, indicating an expected increase in the number of modelers $v s$ only $29 \%$ of small to mid-sized companies indicating an expected increase. Overall, preclinical PK/PD modeling is increasing in demand. Preclinical PK/ PD modelers come from a variety of backgrounds (Fig. 3d). By far, the most common background of preclinical PK/PD modelers is pharmacokinetics/pharmaceutics with all companies having preclinical modelers with this background. The background is likely to be linked to the most common training for scientists working in preclinical Drug Metabolism and Pharmacokinetics (DMPK) departments since many modelers are members of DMPK departments and are closer to the projects and the biological issues (see below).

\section{Organizational Structure}

The results of survey questions focused on gathering information on how preclinical PK/PD modelers are organized are shown in Figs. 4, 5, 6, and 7. The results indicate that, in general, for the majority of the companies, preclinical PK/PD modelers (91\%) are members of a DMPK department/group (Fig. 4). All small to mid-sized companies participating in the survey indicated having PK/PD modelers in DMPK. Surprisingly, two large companies did not report having any preclinical PK/PD modelers in their DMPK groups. In addition to DMPK, a large percentage of pharmaceutical companies indicated having preclinical PK/ PD modelers reside in the clinical pharmacology/translational sciences $(41 \%)$ and modeling and simulation (23\%) groups (Fig. 4). The presence of preclinical modelers in clinical modeling groups is not surprising since clinical modeling groups were likely established earlier than their preclinical counterparts. A lower percentage of large companies had preclinical PK/PD modelers in these two groups external of DMPK compared to small to mid-size companies $(47 \%$ vs $84 \%$ ). In most large companies, preclinical groups (such as DMPK) are likely to be resourced sufficiently such that there is less reliance on groups that traditionally support clinical $\mathrm{PK} / \mathrm{PD}$ to be involved with the preclinical modeling.

Half of the companies that participated in the survey reported having formal $\mathrm{PK} / \mathrm{PD}$ or modeling and simulation (M\&S) group(s) within their organizations (Fig. 5a). The majority of these companies (92\%) having a formal M\&S group(s) reported that these formal $M \& S$ group(s) report into the DMPK group. A smaller percentage of the companies also indicated that the $\mathrm{M} \& \mathrm{~S}$ group(s) reported into clinical pharmacology, translational sciences, and/or quantitative sciences groups (Fig. 5b). For pharmaceutical companies with a formal $\mathrm{PK} / \mathrm{PD}$ or $\mathrm{M} \& \mathrm{~S}$ group, this group is most commonly 


\section{a All Companies $(n=22)$}

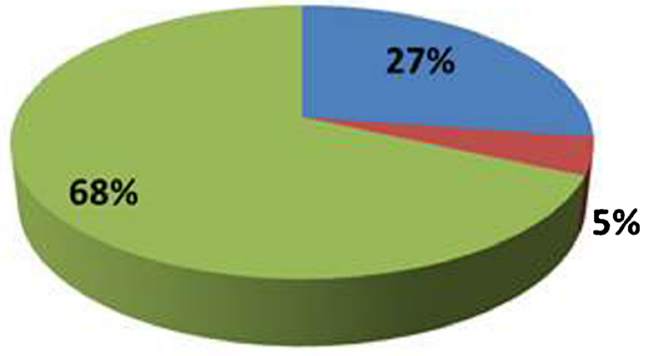

Mostly small molecule

Mostly biologic

Mixed

\section{Small to Mid-Sized Companies $(n=7)$}

b

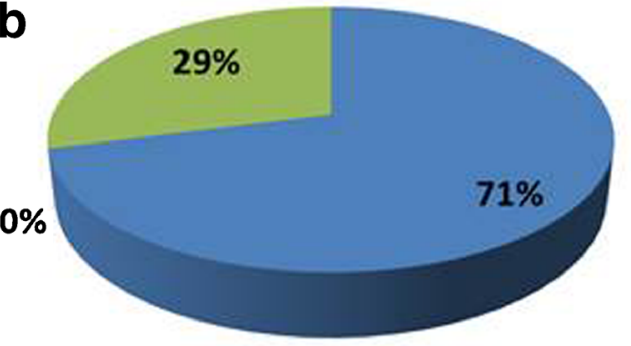

C

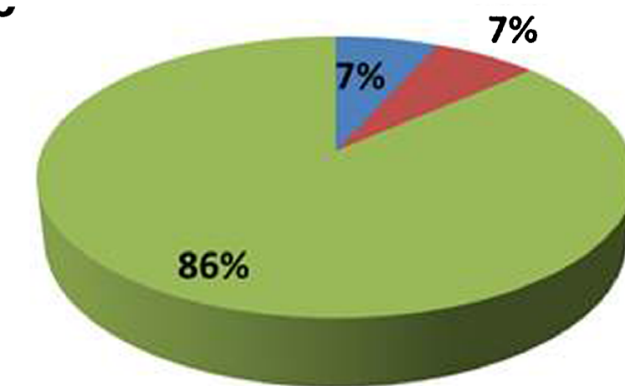

anostly small molecule

Mostly biologic

Mixed

Fig. 2. Pie charts indicating proportion of pharmaceutical companies working on small molecules, biologics, or both small molecules and biologics (mixed). a Pie chart for all companies $(n=22)$ responding to survey, b small to mid-sized companies $(\leq 10,000$ employees; $n=7$ ), and c large companies ( $>10,000$ employees; $n=15$ )

centralized $(\sim 59 \%)$ or organized into groups depending on geographic regions (29\%) (Fig. 6a). This difference in organization may reflect differences in how the drug discovery organizations are arranged with some companies finding more efficiency in pooling modeling resources into one group (global), whereas others prefer closer contact to the "end users" (local). Furthermore, some companies may only be in one geographic region. An examination of trends between larger $v s$ small to mid-sized companies shows that larger companies seem to have a slight preference for a centralized preclinical PK/PD group (67\% for larger companies vs $40 \%$ for small to mid-sized companies).

More companies have preclinical PK/PD modelers that are an integral part of the project team (64\%) rather than acting as a "service provider" (36\%) (Fig. 6b). This partition was independent of company size. This percentage differs from the percentage of companies with formal PK/PD group(s) (Fig. 5a) where one would expect more "service provider" type project support by formal groups. Direct integration of modelers into project team likely increases their effectiveness. Thus, there is likely some integration into discovery project teams even for companies with formal PK/ PD groups.

In general, dedicated preclinical PK/PD modelers are a minority within the companies that responded to the survey (Fig. 7). Only two large companies (9.5\%), both with over 10,000 employees, reported that their preclinical PK/PD modelers were fully dedicated to modeling and simulation work, while the other 19 companies reported that their preclinical PK/PD modelers had other responsibilities beyond providing modeling and simulation support. Out of these 19 companies, 11 indicated that their modelers were also DMPK representatives in project teams. Interestingly, the two companies with dedicated preclinical PK/PD modelers did not report having a formal $\mathrm{PK} / \mathrm{PD}$ or $\mathrm{M} \& \mathrm{~S}$ group to support preclinical $\mathrm{PK} / \mathrm{PD}$ modeling within their organizations. 


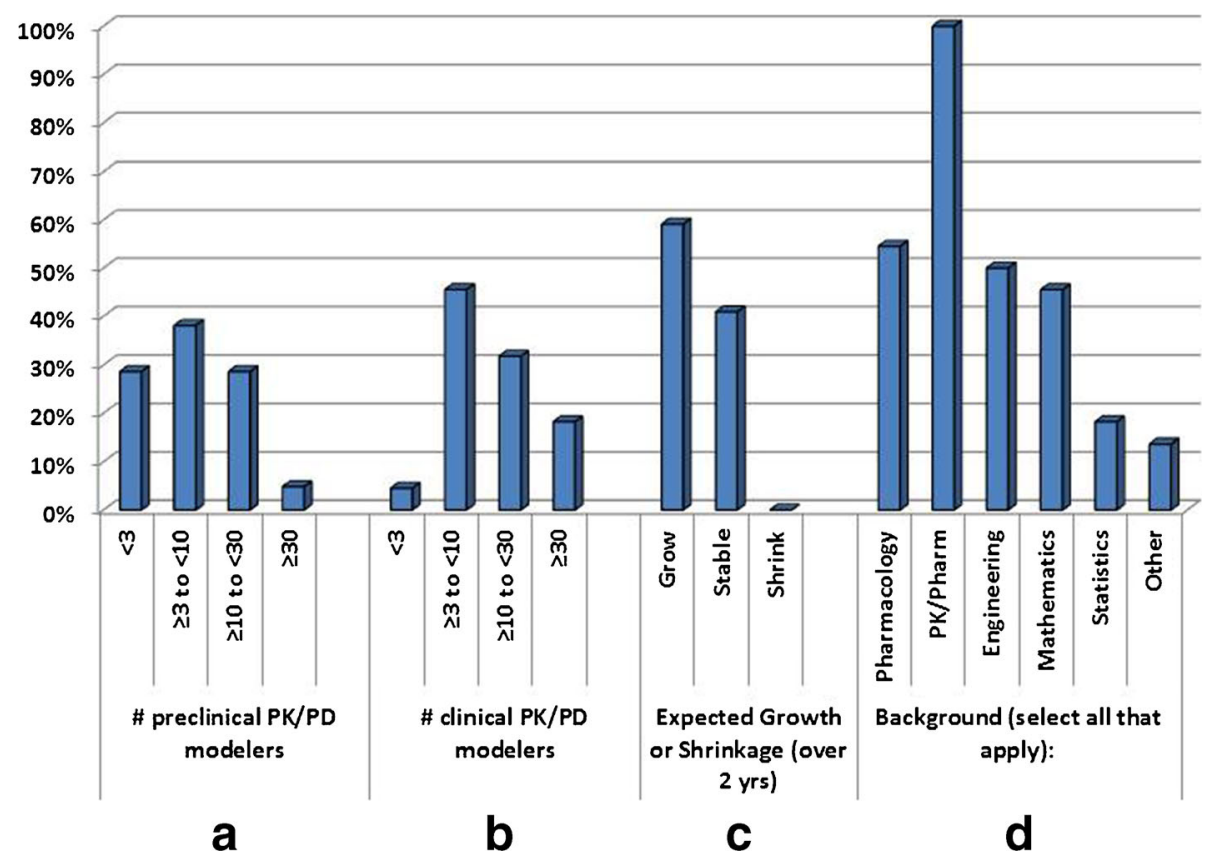

Fig. 3. Bar graphs indicating relative number of preclinical and clinical PK/PD modelers $(\mathbf{a}, \mathbf{b})$, anticipated growth of preclinical PK/PD groups over the next 2 years $\mathbf{c}$, and training background of preclinical PK/PD modelers (d) in pharmaceutical companies responding to the survey. PK/Pharm in Fig. $3 \mathrm{~d}$ is an abbreviation for pharmacokinetics/pharmaceutics

Overall, these results indicate that, in general, preclinical PK/ PD modeling and simulation is not a dedicated activity, but an expected and desired skill for members of the DMPK groups. In most companies (59\%), preclinical PK/PD modelers handle three to four projects at one time (Fig. 7). The number of projects assigned to modelers seems to be independent of company size.

\section{Operating Logistics}

\section{Timing}

The most common response from companies (approximately half) was that preclinical PK/PD modeling and simulation activities were initiated during the "late lead optimization" stage (Table I, question A1). This was true for both large and small to mid-size companies. The timing reflects the stage of drug development where an adequate amount of information is known about a specific molecule to enable preclinical PK/PD analysis. Typically, any modeling efforts occurring earlier than this stage would only be performed on a "tool" or "benchmark" compound if one was available and suitable to characterize in an in vivo $\mathrm{PK} / \mathrm{PD}$ or efficacy model. However, it is fairly common that such a "tool" or "benchmark" molecule does not exist at stages earlier than late lead optimization especially for novel therapeutic targets. The amount of preclinical modeling that occurs during "post-IND" and "clinical development" is expected to be less since clinical biomarker and pilot efficacy data is often available post-IND from clinical trials, and is of greater relevance. In $\sim 18 \%$ of companies, the initiation of preclinical $\mathrm{PK} / \mathrm{PD}$ modeling is dependent on the disease area

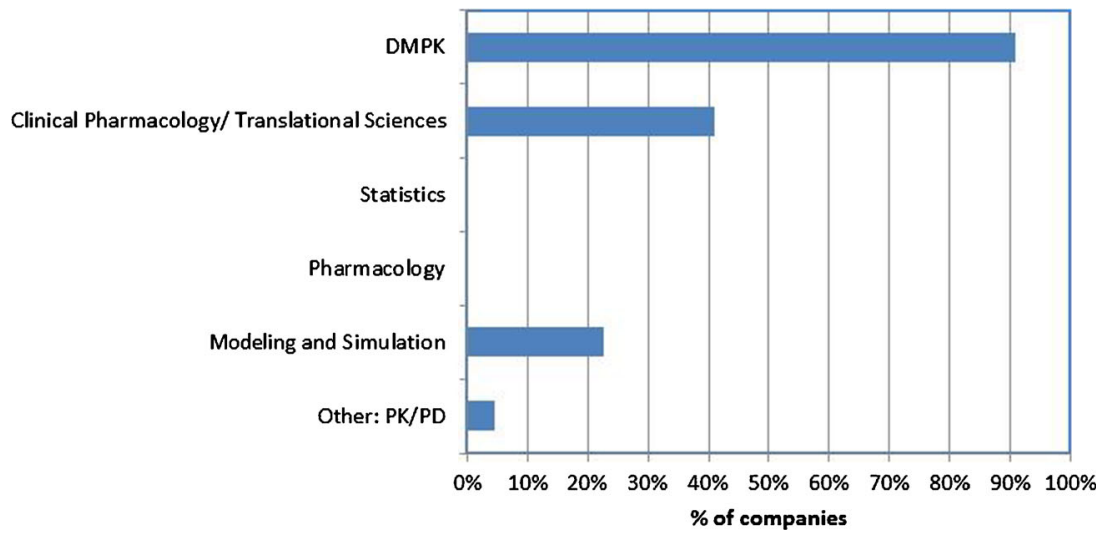

Fig. 4. The group/department where the majority of the preclinical PK/PD modelers reside in for pharmaceutical companies responding to the survey. Responses are expressed in the figure as a percentage of pharmaceutical companies providing a given response. Selection of more than one response was allowed if required 
a

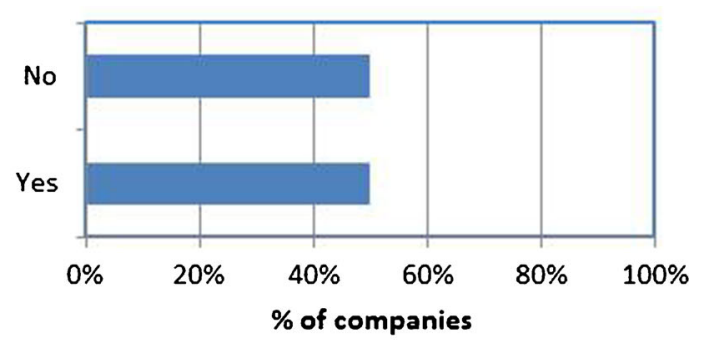

b

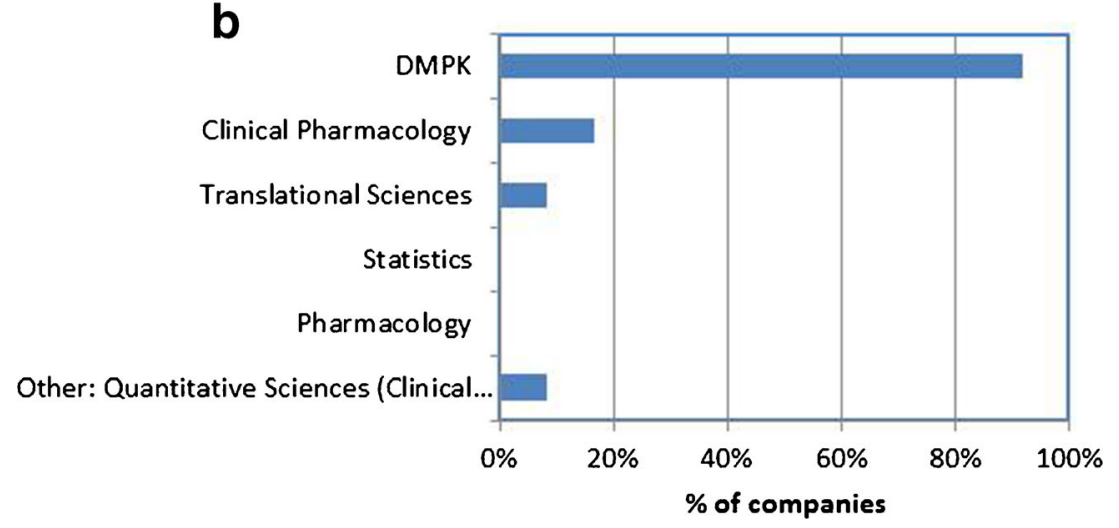

Fig. 5. Bar graphs indicating a the percentage of companies having formal $\mathrm{PK} / \mathrm{PD}$ or modeling and simulation group(s) to support preclinical PK/PD modeling and $\mathbf{b}$ the function/group that this formal PK/PD group reports into. For $\mathbf{b}$, selection of more than one option was allowed if there was more than one group performing preclinical $\mathrm{PK} / \mathrm{PD}$ modeling and simulation

(Table I, question A1). This may be related to differences in the availability and/or suitability of preclinical in vivo efficacy or PK/PD models for various therapeutic areas.

More companies reported that PK/PD modeling was planned ahead of time with input from modelers on study design $(64 \%)$ rather than without input from modelers (36\%) (Table I, question A2). Interestingly, the same percentage of

\section{a}

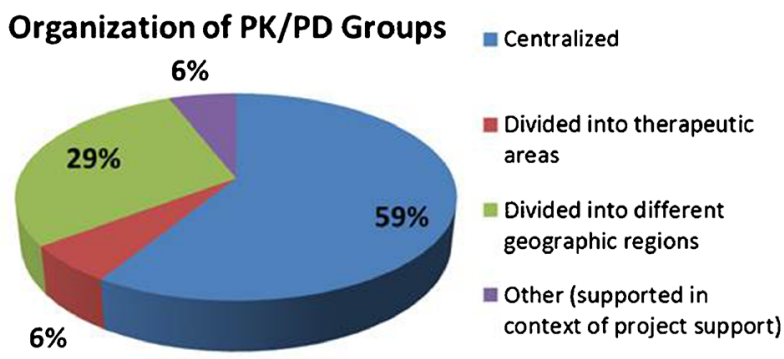

b

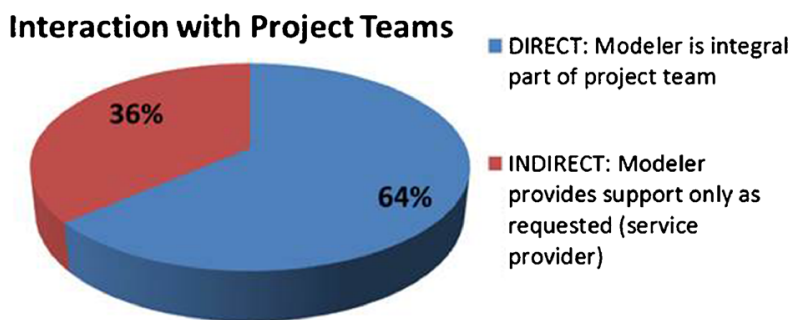

Fig. 6. Pie charts showing the percentage of pharmaceutical companies that participated in survey with a formal PK/PD groups that are either centralized, or divided and $\mathbf{b}$ preclinical PK/PD modelers that are either direct or indirect members of the project teams companies responded that preclinical PK/PD modelers were an integral part of the project team $v s$ being "service providers" (Fig. 6b). Based on personal experiences and observations, the authors felt that the responses to these two questions were linked since a consequence of including modelers into the project teams is that they are aware of and assist in the design of preclinical efficacy studies such that the data that are acquired are suitable to build quantitative PK/PD models.

\section{Standardization and Information Transfer}

A cursory look at preclinical modeling practices suggests that companies in general $(>90 \%)$ are attempting some form of standardization or overlap of practices. In the majority of companies $(58 \%)$, there appears to be some overlap in modeling practices by each particular therapeutic area; however; each therapeutic area can develop their own practices (Table I, question B1). Some individuality of preclinical modeling practices may exist as preclinical biology models for each therapeutic area likely differ in terms of experimental design, biomarkers, and relevance to human disease.

An examination of standardization and transfer of knowledge between preclinical and clinical PK/PD modeling groups suggests that most companies $(>60 \%)$ attempt to ensure that information is transferred as there appears to be either shared databases or transfers/hand offs of information when the drug candidates advance from the preclinical to the clinical space (Table I, question B2). This appears to be an area that requires further development since there is a third of companies $(33 \%)$ where any type of knowledge transfer 


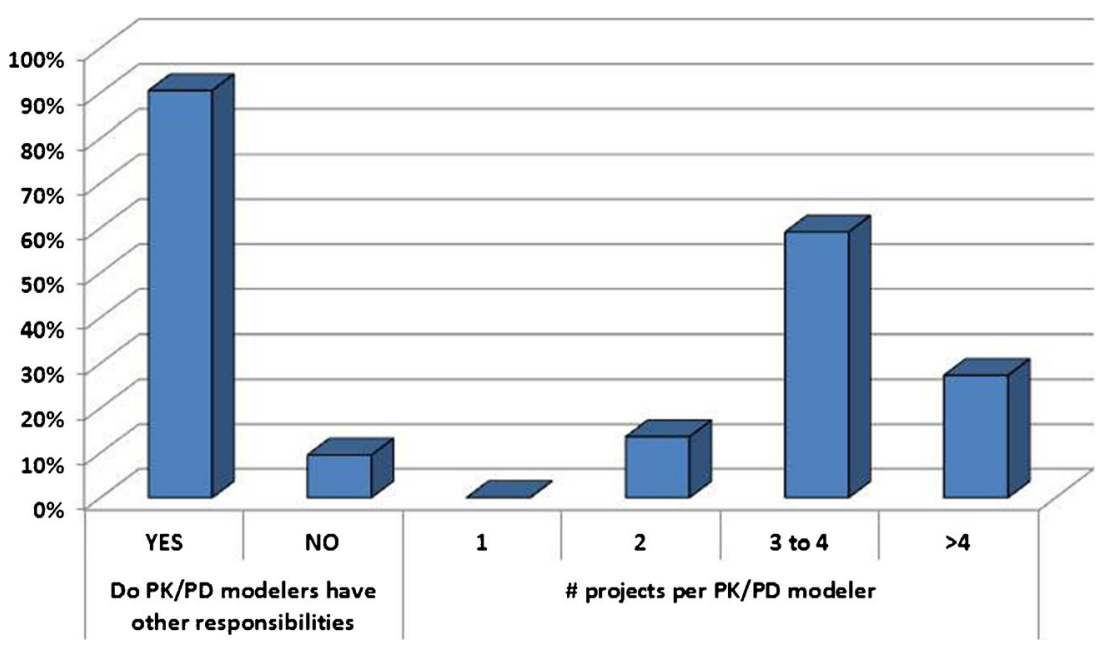

Fig. 7. Bar graphs showing percentage of pharmaceutical companies where preclinical PK/ PD modelers have other responsibilities beyond PK/PD modeling, and the number of projects each modeler typically handles at one time

process does not exist (Table I, question B2). Lack of a formal process for knowledge transfer appears to be more prevalent for small to mid-sized companies (50\%) compared to large companies $(25 \%)$.

\section{Data Source}

Although both in vitro and in vivo data are being utilized for preclinical modeling, the primary dataset for preclinical modeling appears to be derived from in vivo studies $(86 \%)$ (Table I, question C1). Only three companies, all of which were small to mid-size companies, responded that their primary dataset for $\mathrm{PK} / \mathrm{PD}$ modeling was in vitro. Since in vitro studies are in general less costly than in vivo studies, this observation might again be a reflection of a budgetdriven decision than a strategic one. Most of the in vivo data used for preclinical PK/PD modeling is still generated inhouse based on the low percentage of companies responding that data for preclinical PK/PD modeling is mostly generated at CROs (9.1\%) (Table I, question C2). There appeared to be less of a reliance on CROs from large pharmaceutical companies with $60 \%$ of large companies, indicating that data came mostly from studies performed in-house.

Finally, we looked at differences in how the preclinical and clinical modeling groups utilized outsourcing for PK/PD analyses (Table I, questions C3 and C4). Overall, it appears that more outsourcing was performed by the clinical $(\sim 35 \%)$ rather than the preclinical modeling groups $(\sim 13 \%)$. Potentially, there are a number of reasons why this trend exists. First, the nature of modeling clinical data sets may play a role. Modeling of clinical data sets requires more standardization, and often, the analyses are laborious and operate under fixed timelines, and with well-vetted analysis strategies. These characteristics make outsourcing more amenable for clinical PK/PD data analyses. In addition, the establishment of specialized clinical modeling groups likely preceded the establishment of the analogous preclinical modeling groups, leading to a natural establishment of several capable outsource providers. Therefore, the higher amount of outsourcing may be related to how long clinical PK/PD groups have been operating. It may be of interest to see if this trend remains the same over time. No consistent trend in outsourcing was observed when comparing large companies to small and mid-sized companies.

\section{Software}

In order to understand which software is being utilized for preclinical PK/PD modeling, a question was included to survey the prevalence in use of various software packages (Table I, question D1). Phoenix/WinNonlin (100\%), SIMCYP (86\%) and NONMEM (77\%) appear to be the most widely used software packages by scientists in pharmaceutical industry for preclinical PK/PD modeling.

\section{Applications}

Responses to the survey indicate that preclinical PK/PD modeling is used by almost all companies involved with IQ and widely used by most companies $(\sim 68 \%)$ in all therapeutic areas (Table II, question A). Overall, the preclinical PK/PD modelers appear to be quite well integrated with the translational biology/pharmacology groups as preclinical modelers in most companies ( $67 \%)$ have some influence over the study design on preclinical pharmacology studies. The response is consistent with a similar question (Table I, question A2) described in an earlier section of the survey. However, there is room for improvement as modelers in approximately a third of the companies have little or no influence over study design (i.e., selection of either "rarely" or "never" as responses to question B in Table II).

It appears that preclinical PK/PD modeling support is provided to almost all functions that are involved in the drug discovery setting based on the response to question $\mathrm{C}$ in Table II. Preclinical modeling support is mostly provided in the context of a project team $(\sim 91 \%)$, whereas support provided to all other functions appears to be evenly distributed ranging from $41 \%$ (biology) to $68 \%$ (clinical pharmacology and in vivo pharmacology). Approximately $14 \%$ of companies supplied preclinical modeling PK/PD support to other functions that were not specified in the survey response. These functions include medicinal chemistry, 
Table I. Operating Logistics

Questions

A. Timing

A1. At what stage is preclinical PK/PD modeling normally initiated in your company?

A2. Regarding the timing in which preclinical modeling is done, please indicate the option that best applies:

B. Standardization and information transfer

B1. If preclinical PK/PD modeling group is not centralized, are practices the same or different with groups supporting different indications/therapeutic areas?

B2. If the group is divided between preclinical and clinical, how are the models shared?

C. Data source

C1. Indicate the PRIMARY dataset used for the modeling projects in your company

C2. Where is the data generated most of the time?

C3. In your company, what percentage of pre-clinical $\mathrm{PK} / \mathrm{PD}$ modeling is done in-house versus outsourced to modeling CROs?

C4. In your company, what percentage of clinical $\mathrm{PK} / \mathrm{PD}$ modeling is done in-house versus outsourced to modeling CROs?

D. Software

D1. What are the software tools used in your company for preclinical PK/PD modeling (check all that apply):
Answers

Response (\%)

Hit-to-lead

9.1

Late lead optimization $\quad 45.5$

Pre-IND 13.6

Post-IND

Clinical development

0

9.1

Depends on therapeutic area or project $\quad 18.2$

My company does not do this 4.5

Modeling is planned ahead of time, modelers $\quad 63.6$

participate in efficacy study design

Efficacy studies are planned ahead of time, mostly without modeler's input (modeling is opportunistic)

Practices are standardized to all therapeutic areas $\quad 33.3$

There is overlap of some practices, but each $\quad 58.3$

therapeutic area can develop their own practices

Groups are completely independent; practices can vary significantly form one group to another

We have a shared database of models and data

There is an official hand-off of data/databases, models, and reports, which ends the involvement of the preclinical team

There is a gradual hand-off of data/databases, models, and reports, with the preclinical modeler working with the clinical team to keep developing the model for clinical application

No formal process to transfer knowledge from preclinical to clinical; this has generally been a missed opportunity within our organization

In vitro $\quad 13.6$

In vivo $\quad 86.4$

In silico 0

Literature

Other

Data is generated mostly from in-house studies $\quad 45.5$

Data is generated mostly at CROs $\quad 9.1$

Data can be generated either in-house or at $\quad 45.5$

$\mathrm{CRO}$; depends on the project

In-house (\%) 90

Outsourced (\%) 13.3

In-house (\%) $\quad 65.2$

Outsourced (\%) 34.8

Phoenix/WinNonlin $\quad 100$

NONMEM $\quad 77.3$

SAAM II $\quad 31.8$

ADAPT V 22.7

MATLAB $\quad 50.0$

Berkeley Madonna $\quad 40.0$

SIMCYP $\quad 86.4$

GastroPlus $\quad 59.1$

Statistical packages: R, SAS, Splus, etc. $\quad 45.5$

Others $^{a}$ (Mathematica; ACSL-TOX; $\quad 18.2$ MONOLIX; SCIENTIST)

${ }^{a}$ The written responses supplied by those who selected "Others" are shown in the parentheses

pharmaceutics, and imaging. The primary goal of preclinical PK/PD analysis for all companies (100\%) appears to be selection/optimization of doses and/or dose regimens, including prediction of human efficacious doses. This was followed by "comparison with competitor compound" (64\%) and "assess optimal target engagement" (59\%) (Table II, 
Table II. Applications

Question

A. How would you describe the use and application of PK/PD modeling across functional areas in your company?

B. How often does PK/PD modeling inform preclinical pharmacology study design in your company?

C. In your company, to what function do the modelers provide preclinical PK/PD support to.

Please select all that apply.

D. Please indicate the primary goal of the PK/PD modeling in your company (check all that apply).

E. In what therapeutic areas does preclinical PK/PD modeling provide the most support in your organization?

F. In what therapeutic areas does preclinical PK/PD modeling provide the least support in your organization?

G. What therapeutic area has the implementation of preclinical PK/PD modeling been the most impactful?

H. Regarding systems pharmacology, to what extent is it being used in your company?

I. Which departments are actively involved in the use of systems pharmacology? Please indicate all that apply.

J. How often is PBPK analysis combined with a PD measurement (PB/PK/PD)?

\section{Answers}

Response (\%)

Used and applied in all therapeutic areas

68.2

Widely applied but not in all therapeutic areas

Weakly supported; limited to one or two therapeutic areas 4.5

Not used

Always

Frequently

4.5

0

66.7

Rarely

28.8

Never

Biology

In vivo pharmacology $\quad 68.2$

Toxicology $\quad 54.5$

Translational medicine $\quad 59.1$

Clinical pharmacology $\quad 68.2$

Project teams directly $\quad 90.9$

Others (medicinal chemistry; pharmaceutics; imaging) 13.6

Description of the data with a mechanistic/semi-mechanistic $\quad 68.2$ model

Target validation $\quad 18.2$

Triage compounds for advancement $\quad 45.5$

Comparison with competitor compound 63.6

Assess optimal target engagement $\quad 59.1$

Selection/optimization of doses and/or dose 100.0 regimens, including prediction of human doses

Optimal sampling times $\quad 50.0$

Other $^{a}$ : (medicinal chemistry design; aid 18.2

in vivo study design)

Oncology 40.9

Neuroscience $\quad 13.6$

Autoimmune disorders $\quad 0$

Infectious diseases $\quad 4.5$

Cardiovascular 0

Metabolic disorders 13.6

Other $^{a}$ (similar across multiple therapeutic areas; biologics) $\quad 27.3$

Oncology $\quad 10.0$

Neuroscience $\quad 35.0$

Autoimmune disorders $\quad 20.0$

Infectious diseases $\quad 10.0$

Cardiovascular 0

Metabolic disorders $\quad 5.0$

Other $^{a}$ (renal) 20.0

Oncology 38.1

Neuroscience $\quad 14.3$

Autoimmune disorders $\quad 4.8$

Infectious diseases $\quad 9.5$

Cardiovascular 0

Metabolic disorders $\quad 14.3$

Other $^{a}$ (all therapeutic areas; biologics) $\quad 19.0$

Never $\quad 9.1$

Sometimes $\quad 86.4$

Often/always $\quad 4.5$

What is systems pharmacology?

Pharmacology $\quad 38.1$

Biology 14.3

Biomarkers/bioinformatics $\quad 28.6$

DMPK $\quad 52.4$

Statistics $\quad 9.5$

Others $^{a}$ (clinical pharmacology; modeling \&simulation; $\quad 38.1$ computational biology; translational medicine)

$\begin{array}{ll}\text { Never } & 22.7 \\ \text { Sometimes } & 72.7\end{array}$

Often/always $\quad 4.5$

\footnotetext{
${ }^{a}$ The written responses supplied by those who selected "Others" are shown in the parentheses
} 
question D). These responses are consistent with preclinical $\mathrm{PK} / \mathrm{PD}$ modeling being used in the context of a project team since these activities provide very important information used to select and advance drug candidates to the clinic, which is the primary goal of drug discovery project teams.

Of the various therapeutic areas supported by preclinical PK/PD modeling, oncology was clearly most supported ( $41 \%$; Table II, question $\mathrm{E})$. This was followed by neuroscience and metabolic disorders (both $\sim 14 \%$ ). Accordingly, the therapeutic area where preclinical PK/PD modeling showed the most impact was in oncology (38\%) followed by neuroscience $(\sim 14 \%)$ and metabolic disorders $(\sim 14 \%)$ (Table II, question G). Dose and schedule are of critical importance for the development of oncology agents. Therefore, the high use of preclinical PK/PD modeling in oncology is in line with the response from all companies that primary goal of preclinical PK/PD modeling is "selection/optimization of doses and/or dose regimens, including prediction of human doses." The therapeutic area that was least supported by PK/ PD modeling was "neuroscience" at 35\% (Table II, question F). Surprisingly, neuroscience was also among the therapeutic areas where modeling was the most supported and most impactful as discussed above. Based on these responses, it appears that some companies perform preclinical PK/PD modeling using neuroscience data where others are of the view that there is little value gained. The results may also be reflective of the specific CNS disease that each company is working on and the degree of translatability to man of the particular preclinical CNS disease models being used.

In contrast to the wider use of preclinical PK/PD modeling, the majority of the survey participants $(\sim 86 \%)$ indicated systems pharmacology models are only "sometimes" used (Table II, question H). The majority of systems pharmacology users reside in DMPK (52\%), which suggests that responses include the use of commercial software such as SIMCYP and GastroPlus, which are physiologically based pharmacokinetic (PBPK) modeling software often included under the broader systems pharmacology umbrella (Table II, question I). The lower frequency of use of systems pharmacology models compared to preclinical PK/PD models may relate to the larger amounts of compound specific information needed to build a systems pharmacology model as well as the relative "infancy" of the discipline in pharmaceutical industry. Large amounts of compound specific information are often not available early on in the drug discovery process. In addition, information around new biological targets is typically incomplete and thus serves to impede the development and implementation of systems pharmacology models.

It appears that use of hybrid PBPK models with PK/PD components $(\mathrm{PB} / \mathrm{PK} / \mathrm{PD})$ is gaining interest as a large number of companies indicated some use of $\mathrm{PB} / \mathrm{PK} / \mathrm{PD}$ modeling based on the large combined responses of either "sometimes" $(72.7 \%)$ or "often/always" (4.5\%) (Table II, question J). However, the low number of companies selecting "often/ always" indicates that this practice of using PBPK modeling in combination with preclinical PK/PD analysis is not commonplace. In addition, $43 \%$ of the small to mid-size companies indicated that PBPK is never combined with PD measurements, compared to only $13.3 \%$ in larger companies. This can be explained by the fact that PBPK models require a large amount of data and expertise to develop, which may impede its common use with PD measurements, especially in smaller sized companies. In addition, commercial PBPK software are generally more expensive compared to traditional PK/PD software, so this observation may simply reflect the ability of larger companies to afford PBPK software.

\section{Impact/Perspectives}

Generally, it appears that preclinical PK/PD modeling is fairly established and growing in the IQ companies that responded to the survey with all companies viewing preclinical modeling as either "very important" or "somewhat important" (Table III, question A). Larger companies more frequently viewed preclinical modeling as "very important" with approximately double the number of responses from large companies when compared to smaller organizations (53\% vs $28 \%$ ). This slight difference may be due to more resourcing to perform this work in large organizations.

Considering the primary goal of pharmaceutical companies is to discover and develop new medicines, a potential area of growth could be in the application of preclinical PK/ PD modeling with a particular emphasis towards influencing the drug development process. Currently, in only approximately a third of the companies (32\%), preclinical PK/PD modeling appears to "always" impact the decision-making process in drug development (Table III, question B). Approximately 59\% of the companies responded that preclinical modeling sometimes has an impact on decision making and the remaining $9 \%$ of the companies view the data as being largely descriptive in nature and rarely has any influence on decision making. Larger pharmaceutical companies appear to value the use of preclinical PK/PD modeling and its impact/influence on decision making more compared to small to mid-sized companies based on a higher percentage of responses indicating that it "always" impacts the decision making process $(40 \%$ vs $14 \%)$. There appears to be no relationship between therapeutic area that preclinical PK/PD modeling is most impactful and its influence on the decisionmaking process as all companies that replied "always" for question B in Table III found its use to be most impactful across different therapeutic areas (data from responses to question $G$ in Table II). Preclinical PK/PD modeling is on critical path for molecule advancement in more than half of the companies surveyed at $\sim 64 \%$ (Table III, question C). However, this is double the percentage of companies that report $\mathrm{PK} / \mathrm{PD}$ analysis always influencing decision making (Table III, question B). A critical look at how these preclinical PK/PD analyses are used in the decision-making process is warranted in order to improve its effectiveness considering the large number of companies that have this activity on critical path.

An examination of factors impeding the implementation of preclinical PK/PD modeling suggests that multiple factors make implementation difficult (Table III, question D). The factors that half of the companies identified as issues are the inability to "gain support from project/management team" and the lack of "time and resources for continuous training of staff." As many of the modelers reside in DMPK (Fig. 4), it is possible that the preclinical PK/PD modeling efforts grew out of existing DMPK groups. Typically, only advanced or "tool" 
Table III. Impact/Perspectives

\begin{tabular}{|c|c|c|}
\hline Question & Answers & Response (\%) \\
\hline \multirow{3}{*}{$\begin{array}{l}\text { A. What is the general view within your company } \\
\text { on the importance of preclinical PK/PD modeling? }\end{array}$} & Very important & 45.5 \\
\hline & Somewhat important & 54.5 \\
\hline & Not important & 0 \\
\hline \multirow{3}{*}{$\begin{array}{l}\text { B. Does preclinical PK/PD modeling have an impact } \\
\text { on drug development decision-making in your company? }\end{array}$} & Always & 31.8 \\
\hline & Sometimes & 59.1 \\
\hline & Rarely, it is mostly descriptive & 9.1 \\
\hline \multirow{2}{*}{$\begin{array}{l}\text { C. Is preclinical PK/PD on the critical path for } \\
\text { molecule advancement in your organization? }\end{array}$} & Yes & 63.6 \\
\hline & No & 36.4 \\
\hline \multirow{8}{*}{$\begin{array}{l}\text { D. What do you think are the challenges for PK/PD } \\
\text { modeling implementation in your company? } \\
\text { Please select all that apply. }\end{array}$} & Gain support from project/management team & 54.5 \\
\hline & Lack of expertise (trained modelers) & 36.4 \\
\hline & Lack of time to dedicate to modeling projects & 45.5 \\
\hline & Lack of time and resources for continuous training of staff & 54.5 \\
\hline & Lack of appropriate tools (software programs) & 0 \\
\hline & Not enough data available or shared across groups/teams & 45.5 \\
\hline & $\begin{array}{l}\text { Lack of communication across different functions } \\
\text { (e.g., DMPK, Pharmacology, Clin Pharm, Biostats, etc.) } \\
\text { or geographical regions }\end{array}$ & 50.0 \\
\hline & $\begin{array}{l}\text { Other }^{a} \text { (lack of awareness, lack of clear objectives, } \\
\text { retention) }\end{array}$ & 18.2 \\
\hline \multirow{3}{*}{$\begin{array}{l}\text { E. Other than human PK prediction or PK DDI } \\
\text { liability assessment, how often is preclinical PK/PD } \\
\text { modeling included in regulatory submissions such as } \\
\text { IND, IB, and briefing documents in your company? }\end{array}$} & Never & 27.3 \\
\hline & Sometimes, it depends on the project & 54.5 \\
\hline & It is often included & 18.2 \\
\hline \multirow{3}{*}{$\begin{array}{l}\text { F. Regarding regulatory submissions, is there an increasing } \\
\text { trend to date, or expected over the coming years? }\end{array}$} & Certainly, yes & 54.5 \\
\hline & Maybe, not sure & 45.5 \\
\hline & No & 0 \\
\hline \multirow{4}{*}{$\begin{array}{l}\text { G. Is preclinical PK/PD modeling used to inform the } \\
\text { phase } 2 \text { POC study design in your company? }\end{array}$} & Always & 13.6 \\
\hline & Sometimes, if the models are available & 54.5 \\
\hline & Not very often, even when models are available & 22.7 \\
\hline & Never & 9.1 \\
\hline \multirow{2}{*}{$\begin{array}{l}\text { H. Have you personally seen PK/PD modeling have an } \\
\text { impact in drug development? }\end{array}$} & Yes & 86.4 \\
\hline & No & 13.6 \\
\hline \multirow{2}{*}{$\begin{array}{l}\text { I. Has your company had interactions with the FDA, } \\
\text { EMA, or other regulatory agency regarding preclinical } \\
\text { PK/PD, PBPK, and PB/PK/PD models? }\end{array}$} & Yes & 47.6 \\
\hline & No & 52.4 \\
\hline \multirow{5}{*}{$\begin{array}{l}\text { J. In what phase of drug development has interaction } \\
\text { with regulatory agencies happened using preclinical } \\
\text { PK/PD, PBPK, and PB/PK/PD models? }\end{array}$} & Pre-IND & 10.0 \\
\hline & Prior to phase 2 & 50.0 \\
\hline & End of phase 2 meeting & 10.0 \\
\hline & Briefing Document & 0 \\
\hline & Other $^{a}$ (interactions occurred in more than 1 phase) & 30.0 \\
\hline
\end{tabular}

${ }^{a}$ The written responses supplied by those who selected "Others" are shown in the parentheses

compounds have enough existing data to perform modeling analysis. As such, preclinical modeling may not be a regular activity on projects, and therefore, obtaining support from the project team and management to get adequate resource to perform such analysis may be prohibitive.

On the regulatory front, it appears that preclinical PK/ PD modeling is increasingly being included in regulatory packages, with approximately $73 \%$ of companies including these analyses in regulatory submissions at some point for particular projects (Table III, question E). Inclusion of preclinical PK/PD modeling in regulatory submissions appears more prominent in larger compared to smaller companies, with only $13 \%$ of large companies never including these data in regulatory packages compared to $57 \%$ for smaller companies. When asked whether inclusion of preclinical PK/ PD modeling as a trend would increase in regulatory submissions, companies surveyed were evenly split between answering "yes, certainly" and "maybe, not sure" (Table III, question F). The use of preclinical PK/PD modeling to inform phase II POC study design appears to be company and likely therapeutic area dependent (Table III, question G). Only $14 \%$ of companies use preclinical PK/PD modeling to "always" inform phase II proof of concept (POC) study design. For other companies, its use for phase II appears to be inconsistent and likely dependent on the particular project as the majority of the surveyed companies ( $77 \%$ combined) responded with "sometimes, if the models are available" or "not very often, even when models are available." Finally, the majority of companies (86.4\%) have seen preclinical PK/PD modeling have an impact in drug development (Table III, question $\mathrm{H}$ ). Approximately half of the companies surveyed ( $48 \%$ ) have had regulatory interactions with the FDA regarding preclinical modeling of some form (Table III, question I). Most of these interactions occurred prior to phase II likely because preclinical data typically becomes less 
informative as more and more clinical data are collected (Table III, question J).

\section{CONCLUSION}

Preclinical PK/PD modeling is widely used in pharmaceutical industry with the most common goal of these analyses being to enable the selection/optimization of human doses and/or dose regimens, including prediction of human efficacious doses. However, the consistent use of more complex models including systems pharmacology and hybrid physiologically based pharmacokinetic models with $\mathrm{PK} / \mathrm{PD}$ components was less common compared to traditional PK/PD models. Preclinical PK/PD analysis is increasingly being included in regulatory submissions with $\sim 73 \%$ of companies including these data to some degree. Most companies have seen impact of these preclinical analyses in drug development. The continued growth of preclinical PK/PD modeling groups in pharmaceutical industry is necessary to establish the required resources and skills to further expand the use of preclinical PK/PD modeling in an impactful manner.

\section{ACKNOWLEDGMENTS}

The authors thank Cornelis Hop for his support, feedback on survey questions, and facilitation of the survey. The authors also thank the IQ Drug Metabolism Leadership Group for their support of this survey.

\section{REFERENCES}

1. Arrowsmith J. Trial watch: phase II failures: 2008-2010. Nat Rev Drug Discov. 2011;10:328-9. doi:10.1038/nrd3439.

2. Arrowsmith J. Trial watch: phase III and submission failures: 2007-2010. Nat Rev Drug Discov. 2011;10:87. doi:10.1038/ $\operatorname{nrd} 3375$.

3. Chang C, Byon W, Lu Y, Jacobsen LK, Badura LL, SawantBasak A, et al. Quantitative PK-PD model-based translational pharmacology of a novel kappa opioid receptor antagonist between rats and humans. AAPS J. 2011;13:565-75. doi:10.1208/s12248-011-9296-3.

4. Heimbach T, Gollen R, He H. Practical anticipation of human efficacious doses and pharmacokinetics using preclinical in vitro and in vivo data. In: Wang J, Urban L, editors. Predictive ADMET: Integrated approaches in drug discovery and development. Wiley Inc.; 2014. p. 319-352.

5. Jones RD, Jones HM, Rowland M, Gibson CR, Yates JW, Chien JY, et al. PhRMA CPCDC initiative on predictive models of human pharmacokinetics, part 2: comparative assessment of prediction methods of human volume of distribution. J Pharm Sci. 2011;100:4074-89.

6. Kola I, Landis J. Can the pharmaceutical industry reduce attrition rates? Nat Rev Drug Discov. 2004;3:711-5.
7. Liu L, Di Paolo J, Barbosa J, Rong H, Reif K, Wong H. Antiarthritis effect of a novel Bruton's tyrosine kinase inhibitor in Rat collagen-induced arthritis and mechanism-based pharmacokinetic/pharmacodynamic modeling: relationships between inhibition of BTK phosphorylation and efficacy. J Pharmacol Exp Ther. 2011;338:154-63. doi:10.1124/ jpet.111.181545.

8. Maurer TS, Ghosh A, Haddish-Berhane N, Sawant-Basak A, Boustany-Kari CM, She L, et al. Pharmacodynamic model of sodium-glucose transporter 2 (SGLT2) inhibition: implications for quantitative translational pharmacology. AAPS J. 2011;13:576-84. doi:10.1208/s12248-011-9297-2.

9. Obach RS, Baxter JG, Liston TE, Silber BM, Jones BC, MacIntyre $\mathrm{F}$, et al. The prediction of human pharmacokinetic parameters from preclinical and in vitro metabolism data. J Pharmacol Exp Ther. 1997;283:46-58.

10. Ring BJ, Chien JY, Adkison KK, Jones HM, Rowland M, Jones $\mathrm{RD}$, et al. PhRMA CPCDC initiative on predictive models of human pharmacokinetics, part 3: comparative assessement of prediction methods of human clearance. J Pharm Sci. 2011;100:4090-110.

11. Salphati L, Wong H, Belvin M, Edgar KA, Prior WW, Sampath $\mathrm{D}$, et al. Pharmacokinetic-pharmacodynamic modeling of tumor growth inhibition and biomarker modulation by the novel PI3K inhibitor 2-(1H-indazol-4-yl)-6-(4-methanesulfonyl-piperazin-1ylmethyl)-4-morpholin-4-yl-thieno[3,2-d]pyrimidine (GDC0941). Drug Metab Dispos. 2010;38:1436-42. doi:10.1124/ dmd.110.032912.

12. Vlasakakis G, Comets E, Keunecke A, Gueorguieva I, Magni P, Terranova $\mathrm{N}$, et al. White paper: landscape on technical and conceptual requirements and competence framework in drug/disease modeling and simulation. CPT Pharmacometrics Syst Pharmacol. 2013;2:e40. doi:10.1038/psp.2013.16.

13. Ward KW, Proksch JW, Levy MA, Smith BR. Development of an in vivo preclinical screen model to estimate absorption and bioavailability of xenobiotics. Drug Metab Dispos. 2001;29:82-8.

14. Wong H, Grossman SJ, Bai SA, Diamond S, Wright MR, Grace Jr JE, et al. The chimpanzee (Pan troglodytes) as a pharmacokinetic model for selection of drug candidates: model characterization and application. Drug Metab Dispos. 2004;32:1359-69.

15. Wong H, Choo EF, Alicke B, Ding X, La H, McNamara E, et al. Anti-tumor activity of targeted and cytotoxic agents in murine subcutaneous tumor models correlates with clinical response. Clin Cancer Res. 2012;18:3846-55. doi:10.1158/1078-0432.CCR12-0738.

16. Wong H, Vernillet L, Peterson A, Ware JA, Lee L, Martini J-F, et al. Bridging the Gap between preclinical and clinical studies using PK-PD modeling: an analysis of GDC-0973, a MEK inhibitor. Clin Cancer Res. 2012;18:3090-9. doi:10.1158/10780432.CCR-12-0445.

17. Yamazaki S, Vicini P, Shen Z, Zou HY, Lee J, Li Q, et al. Pharmacokinetic/pharmacodynamic modeling of crizotinib for anaplastic lymphoma kinase inhibition and antitumor efficacy in human tumor xenograft mouse models. J Pharmacol Exp Ther. 2012;340:549-57.

18. Zager MG, Kozminski K, Pascual B, Ogilvie KM, Sun S. Preclinical PK/PD modeling and human efficacious dose projection for a glucokinase activator in the treatment of diabetes. J Pharmacokinet Pharmacodyn. 2014;41:127-39. doi:10.1007/ s10928-014-9351-7. 